\title{
An Investigation of the Impacts of Covid-19 Pandemic Spread on Nurses Fatigue: An Integrated Literature Review
}

\author{
Mahmoud Al-Masaeed, Mansour Al-Soud, ETAF ALKHLAIFAT, Rawan ALSABABHA, \\ Khaldoun Ismail, Nezar Alhabashneh, Sohayb ABU TAPANJEH, Muhammad Alqudah, Irniza \\ Binti Rasdi
}

\begin{abstract}
Study Aim: The integrated review evaluate the impacts of Covid-19 on nurses' fatigue and well-being. The significance was to establish how the pandemic rise and spread have impacted nurses' fatigue prevalence and the practical mitigation strategies, both present and in the foreseeable future.

Research Methodology: An online search on three databases, Scopus, MEDLINE, and CINAHL, was conducted. An expansion strategy using the keywords fatigue, covid-19, and nurses was used. The inclusion criteria were publications in a nursing journals, the publication year 2020, and the availability or publications written in English language. The GRADE model helped drop the less relevant studies.

Findings and Analysis: A total of 47 articles were established. Through the integrated review exclusion criteria, 16 remained as applicable in the analysis. The findings led to the emergence of three key themes, namely (i) rising nursing fatigue levels, (ii) pre-disposition to mental fatigue for nurses with previous mental health complications, and (iii) the need for post-Covid-19 pandemic mental fatigue mitigation strategies. The integrated review implications are the consideration of nurses with pre-existing mental health issues against their deployment as front-line workers. Additionally, the need for long term mental fatigue mitigation strategies is emphasized.

Conclusion: The integrated literature review demonstrates the rising cases of nurses' fatigue amidst the Covid-19 pandemic. Although the pandemic has increased fatigue, the causative/trigger factors presided over the pandemic. The solution and mitigation strategies should be long term and focused on the post-pandemic period.
\end{abstract}

Index Terms - nurses, fatigue, Covid-19 impact, prevalence.

Mahmoud Al-Masaeed, Researcher, RN and a PhD candidate at School of Nursing and Midwifery, Faculty of Health and Medicine, University of Newcastle Australia and University Putra Malaysia.

Mansour Al-Soud, RN, MSc, Master of Advanced Nursing "Education major \& Acute care nursing sub-major" at Faculty of Health, University of Technology, Sydney.

ETAF ALKHLAIFAT, Researcher, $\mathrm{PhD}$ candidate in Linguistics at School of Humanities and Communication Arts, Western Sydney University. Rawan ALSABABHA, PhD Candidate, School of Nursing \& Midwifery, Western Sydney University.

Khaldoun Ismail, $\mathrm{PhD}$ Candidate, School of Nursing \& Midwifery,

Western Sydney University.

Nezar Alhabashneh, RN, MSc, Master of Nursing at School of Nursing \& Midwifery, Western Sydney University.

Sohayb ABU TAPANJEH, RN and a PhD candidate at School of Nursing \& Midwifery, University of Newcastle Australia.

Dr. Muhammad Alqudah, Lecturer at Faculty of Health and Medicine, University of Newcastle Australia.

Assoc/Prof Irniza Binti Rasdi, Senior lecturer at Department of

Environmental and Occupational Health, Head of Occupational Health Unit Department of Environmental and Occupational Health, Faculty of Medicine and Health Sciences, Universiti Putra Malaysia.

\section{INTRODUCTION}

The covid-19 was first reported in Wuhan, China, in late 2019. With the global interconnectedness such as ease of movement and international trade, nations were on high alert on the virus's probability. Despite efforts to contain the spread of the virus within Wuhan, such as through lockdown and fumigations, within three months, nations across Asia and Europe like South Korea, Spain, Italy, and the UK, among others, recorded their first Covid-19 cases (Blake, Bermingham, Johnson and Tabner, 2020; Ruktanonchai, Floyd, Lai, Ruktanonchai, Sadilek, Rente-Lourenco, Ben, Carioli, Gwinn, Steele and Prosper, 2020; Mogi and Spijker, 2020). Among the deployed health front-line workers charged with handling, managing, and caring for the infected patients were nurses. As part of the front-line workers, their work patterns and responsibilities have been impacted. By Mid-March, the World Health Organization (WHO) declared the disease as a global pandemic, elevating it to a worldwide health and economic concern issue for all nations (Blake, Bermingham, Johnson and Tabner, 2020). Despite efforts by countries to contain the diseases spread and the success in reducing overload on the healthcare systems, the high prevalence and spread rates have impacted on the nursing profession work context. Nurses' roles and working timeline have greatly been impacted. The implications extend beyond the direct health concerns (Fowler and Wholeben, 2020; Harrison, 2020). One of the affected categories under the healthcare sector is the nurses. The unique nature of the global world today created a new dimension in the illness. One such included a high prevalence rate globally and the ease and swiftness with which the infections spread from the epicenter in Wuhan China, to new other centers such as Italy in Europe and other global countries. The nurses have been affected due to a change in their workload and professional responsibilities changes (Al-Masaeed, Al-Motiry, Alsababha, Alqudah and Ismail, 2020, p.35). Mental stability is one of the basic factors impacting the nurses' fatigue level and extent of the emerging stress handling and mitigation capabilities (Fowler and Wholeben, 2020). Their mental health, fatigue levels, and coping strategies, and preparedness have been put to the test.

The analysis develops a critical and integrated literature analysis concerning the pandemic implications for nurses. The focus is on evaluating how the fatigue levels and 
prevalence have changed since the pandemic rise. Additionally, the analysis investigates nurses' psychological/mental well-being, especially the front-line workers amidst the pandemic. Finally, it evaluates applicable long-term strategies to address the pandemic's indirect and unexpected impacts among nurses.

\section{MATERIALS AND METHODS}

The review focuses on the impacts of pandemic handling and spread on the nursing profession. This included evaluating the existing data, predominantly developed since December 2019, when the Covid-19 pandemic was officially reported in China. The review included information and data search online from peer-reviewed articles. The focus was on nursing-related journals and publications on fatigue, mental health, and infectious diseases. The search had vital words use. The keywords used in the analysis were "Covid-19 impact," "fatigue," and "nurse." The search process applied inclusion criteria to ensure that the study findings were relevant to the review aims and objectives. The study search was that the articles should have been published in the English language or has an English language translated version. The others included publications that have been published in a peer-reviewed nursing journal, published after December 2019, and available in full article PDF format (Ho, 2008; Kohtala, 2015). Based on the established search criteria, the analysis searched data from three databases: Scopus, MEDLINE, and CINAHL. The search criteria yielded enough literature with Scopus (22), MEDLINE (11), and CINAHL (14).

In the obtained findings, 14 duplications were eliminated. Further, a critical examination of the articles established that some articles (10), although they were obtained within the search parameters, they had no direct correlation with the study findings and were not directly relevant to the study aim. Finally, the articles obtained were analyzed based on their perceived accuracy and applicability. The GRADE model was applied. The model investigates literature and information based on the elements of accuracy, consistency, and risk of errors (Kessels and Guyatt, 2014). The ranking on the model varies from high through moderate, low, and very low. The scale determines the applicability of an article and literature. A minimum moderate ranking indicates relevance and aims synchronization between an article and a study aims and objectives. Thus, the moderate ranking was applied as a threshold for analyzing the findings. This excluded an additional 8 articles (Allen, Chen, Willson and Hughes, 2009, Schunemann, Murad Li, Brignardello-Petersen, Singh, Kessels and Guyatt, 2014). The search process concluded that 15 articles were applicable and fitted the study methodology search process. An evidence table (appendix 1) was developed for the appropriate 15 studies. These articles were used in the study analysis.

\section{RESULTS AND FINDINGS}

The review findings demonstrated that there are rising concerns for nurse s health and well-being. The initial studies on the topic indicated that as the Covid-19 emerged in China and was expected to spread globally, the focus was placed on medical equipment availability (Pillai, Siddika, Apu and Kabir, 2020). Nations and governments focused on ensuring that there were enough medical facilities and equipment. Some of the initial actions included creating isolation centers and revamping the testing systems. Some of the successful contexts, such as South Korea, testing, tracking, and tracing systems, were prioritized. Despite their roles in mitigating and handling the diseases spread, the actions were insufficient (Shim, Tariq, Choi, Lee and Chowell, 2020). The focus on human resources fact has emerged as a new area of research. Nurses serve as a human resource base in providing human care to patients at all levels and units in the healthcare industry. Focusing on their well-being has a ripple effect on nursing care quality. The nurses are a critical part of caring and tendering for Covid-19 patients.. The analysis led to the emergence of three thematic findings. They were (i) Covid-19 has increased nurses exposure to fatigue, (ii) Nurses with pre-existing mental conditions are predisposed, and (iii) besides the short term, during the pandemic, coping strategies, long term post-pandemic strategies should be considered.

\section{A. Nurses fatigue Exposure}

Many studies indicate a high nurse exposure rate to fatigue (Liu, Luo, Haase, Guo, Wang, Liu, Xia, Liu, Yang and Yang, 2020; Yifan, Ying, Chunhong, Jing, Rong, Zhenyu, Zejuan and Peihung, 2020). The nurses' exposure to fatigue has significantly increased firstly, due to the rise in workload and the number of patients served. With the pandemic emergence, nations, including those studied like China, Spain, Italy, and the USA, all had a surge in hospital admissions for Covid-19 patients. This surge negatively impacted the nurses-patients ratio, thus increased the nurses fatigue levels (Hertel 2020; Giusti, Pedroli, D'Aniello, Badiale, Pietrabissa, Manna, Badiale, Riva, Castelnuovo and Molinari, 2020). When nurses are fatigued, exposed, and quarantined, there emerges a shortage in skills across the profession. Finally, nurse fatigue emerged due to the rising number of infected healthcare workers, among them nurses. With increased exposure and infections, there has been a decline in the number of front-line nurse available to care for the Covid-19 patients on the front-line (Bennett, James and Kelly, 2020; Giusti, Pedroli, D'Aniello, Badiale, Pietrabissa, Manna, Badiale, Riva, Castelnuovo and Molinari, 2020). The infections spread from not only the front-line nurse to include the other ordinary ward nurses. These increased rates lowered the number of available nurses. Consequently, a combination of the variables on the lack of enough skills to handle infectious diseases and nurses infections, increased the nurses' potential fatigue levels (Yifan, Ying, Chunhong, Jing, Rong, Zhenyu, Zejuan and Peihung, 2020; Hertel, 2020). Changing care context increases nursing fatigue. There is a high level of compassion fatigue among nurses (Dean and Gentry, 2020). Compassion fatigue emerges when there is a decline in nurses' empathy on patients due to prolonged exposure to suffering and the fear of emotional connection with the patients for the fear of emotional hurt when such patients die. With Covid-19, nurses have minimal capability for physical connections with their patients. This emerges as a result of the 2020 March WHO guidelines on social distancing, general hygiene on handwashing and wearing 
masks in public. For the caregivers, this has led to compassion fatigue as the nurses are unable to show empathy and sympathy to their patients even the non-Covid-19 patients in the manner they are accustomed to in the past (Fowler and Wholeben, 2020; Harrison, 2020).

\section{B. Pre-disposed Nurses Categories to Mental Fatigue and Mitigation Strategies}

The second finding was on the exposure rates among nurses based on their mental health and its impact on their well-being. One of the leading conclusions is acknowledging that the front-line nurses had a higher rate of exposure than their peers working in non-Covid-19 patient departments (Hu, Wang, Hu and Fang, 2020; Hertel 2020). This is a finding supported by the high number of infections and fatigue among the nurses' categories. An additionally pre-disposed category is a nurse with pre-existing mental conditions such as ACE, and traumatic experiences. Conditions such as past work-related trauma and personal childhood related traumatic experiences pre-disposed the nurses to mental fatigue. Results demonstrated that nurses with exposures such as ACE, mental illness, depression, and frustrations were increasingly likely to experience fatigue and health complications than their peers (Fowler and Wholeben, 2020; Harrison, 2020). The Dean and Gentry (2020) study finding was on coping strategies. Researchers suggested the creation of mitigation strategies for those experiencing fatigue including relief from work, support and medical care for the infected, and personal protective equipment's (PPEs) for the nurses, among others (Sun, Wei, Shi, Jiao, Song, Ma, Wang, Wang, Wang, You and Liu, 2020; Dean and Gentry, 2020). . Findings demonstrated that the implications of the Covid-19 pandemic on nurses, especially on the nurses' mental health, are long term and bound to post after the pandemic is controlled. Thus, recommendations emerged on crating long term coping strategies such as the changing of the nurses' educational system, training and development, as well as the provision of psychological and mental support system for all the front-line nurses (Bennett, James and Kelly, 2020; Yifan, Ying, Chunhong, Jing, Rong, Zhenyu, Zejuan and Peihung, 2020; Giusti, Pedroli, D'Aniello, Badiale, Pietrabissa, Manna, Badiale, Riva, Castelnuovo and Molinari, 2020).

\section{DISCUSSION}

By reviewing the findings critics point out that the significant of nursing fatigue, nurses personalized care, and the urgent need for nurses support system.

\section{a) Increase in Nurses Fatigue Levels}

The findings demonstrate of the prevalence of nurses' fatigue levels due to a high exposure rate to changing work dynamics amidst the Covid-19 pandemic. Previous studies on nursing across the world demonstrated a high prevalence index of nursing fatigue (Alharbi, Jackson and Usher, 2020; Yifan, Ying, Chunhong, Jing, Rong, Zhenyu, Zejuan and Peihung, 2020). Among the previously listed causes of fatigue were nurse staff shortages, workload quantity and limitations, and shortage of skills in handling specific tasks and responsibilities. These conditions that were already pre-existing have been increased in the wake of the Covid-19 pandemic. Under the Covid-19 pandemic, a number of contextual factors have changed that can be linked to a high fatigue index. First, with the pandemic, there was an increased demand for nursing and patient care services (Sun, Wei, Shi, Jiao, Song, Ma, Wang, Wang, Wang, You and Liu, 2020; Dean and Gentry, 2020). As the pandemic ranged across nations from China to other countries such as South Korea and then in European nations including Italy, the UK, Spain, and even in the USA, there was a rising demand for front-line nurses (Ruktanonchai, Floyd, Lai, Ruktanonchai, Sadilek, Rente-Lourenco, Ben, Carioli, Gwinn, Steele and Prosper, 2020; Mogi and Spijker, 2020; Pillai, Siddika, Apu and Kabir, 2020). These were nurses charged with caring for the infected and managing the isolation enters for those exposed and under quarantine. Consequently, this sharp spike in cases such as in Italy increased the nurses' workload (Cucinotta and Vanelli, 2020; Mogi and Spijker, 2020).

The strain on workload added to the previous workload challenges exposed the nurses to a high degree of physical, acute, and even chronic fatigue risks, such as back pains and memory loss. A second contextual dimension emerged as the spread and pattern of infection changed. Towards the third month of the pandemic outbreak (February-March 2020), infections on healthcare workers were on the rise which meant increased isolation and quarantine. Concerns were raised on the rising infections and death rate of the healthcare workers. This included the front-line nurses directly caring for and managing the Covid-19 patients.

Consequently, this led to a declining number of available trained and experienced front-line workers who could handle infectious diseases. As a result, the healthcare industry, especially the nurses' professionals who remained front-line workers, faced a rising workload (Hertel 2020; Giusti, Pedroli, D'Aniello, Badiale, Pietrabissa, Manna, Badiale, Riva, Castelnuovo and Molinari, 2020). This is in addition to the inclusion of previously non-experienced nurses in managing and handling infectious diseases patients (Sun, Wei, Shi, Jiao, Song, Ma, Wang, Wang, Wang, You and Liu, 2020; Liu, Luo, Haase, Guo, Wang, Liu, Xia, Liu, Yang and Yang, 2020). The implications of the workforce structure change have been increased physical and acute fatigue (for the front-line nurses remaining) and a rising risk of mental fatigue for nurses without prior experience in managing ad caring for patients with infectious diseases.

\section{b) Special Focus on Nurses with Pre-existing Mental Health Conditions}

Although the findings demonstrate a high level of mental fatigue and risk on psychological well-being for most nurses, a further review and evaluation of the findings indicate variances. While mental fatigue levels have increased across the workforce, the incidences are higher among nurses with pre-existing mental illnesses and conditions such as Traumatic experiences and an adverse childhood experience (ACE) (Pfefferbaum and North, 2020; Spoorthy, Pratapa and Mahant, 2020; Di Tella, Romeo, Benfante and Castelli, 2020). The findings indicate that a high instability and risk of mental fatigue is increased by the lack of complete awareness and, in instances, misinformation. There was minimal 
understanding of the exposure risks and pre-disposing conditions (Greenberg, Docherty, Gnanapragasam and Wessely, 2020). The constant threat of infection among the nurses exposes them to mental instability. Healthcare workers, including nurses, with an existing mental illness, have their recovery and stability plan hedged on operating in a predictable and stable environment. The fear of the unknown should be reduced as much as possible if they remain mentally stable (Nelson and Lee-Winn, 2020; Ng, De Deyn, Lim, Chan and Yeo, 2020).

The findings demonstrate the need for nurses mental health consideration when staffing nurses in handling Covid-19 related cases. Nurses with a stable mental health record are considered for front-line duties.. Although with skills and experience in handling infectious disease patients, nurses with pre-existing mental conditions should be limited on the front-line areas as much as possible. Their mental instability and health risks could endanger their health and that of their patients and families (Spoorthy, Pratapa and Mahant, 2020; Shah, Kamrai, Mekala, Mann, Desai and Patel, 2020). With increased mental nervousness and instability, the nurses are exposed to risks such as medical errors, low and slow response capabilities, and inefficiencies, resulting from mental and chronic fatigue cases in the long-run period.

\section{c) Create Long Term Nurses Mental Health Support} systems

The final emerging theme in the analysis is the creation and establishment of long-term support systems. The findings illustrate that the current focus has been on establishing short-term fatigue mitigation strategies and systems. For instance, there has been a drive to include the available nursing professionals, including volunteer activities by nursing students' and those who had retired or exited the profession but still are in a professionally fit capability (Bennett, James and Kelly, 2020; Blake, Bermingham, Johnson and Tabner, 2020). Other strategies to address nurses mental health and stability have included the provision of PPEs and information and awareness creation among the healthcare professionals, key among them nurses. Although the measures are effective in relieving nurse in the short-term period, it is clear that the Covid-19 pandemic implications will outlive the pandemic. With the projections of the pandemic decline and control with vaccine development, it is clear that the fatigue and the implications on the nursing profession will outlive the pandemic challenges. Some of the long-lasting implications include mental fatigue and changes in the nurses' perspective and relations with peers and patients. This brings to the fore the need to redefine the nursing professional training and skills development systems (Blake, Bermingham, Johnson and Tabner, 2020). It is imperative to incorporate the nurses' mental well-being as a core part of the training process in the future.

Also, the focus should be placed on creating long term fatigue mitigation strategies and systems. Fundamentally, there should be awareness generation on individual level mitigation strategies such as creating an exercise regime among the nurses (Yifan, Ying, Chunhong, Jing, Rong, Zhenyu, Zejuan and Peihung, 2020; Giusti, Pedroli, D'Aniello, Badiale, Pietrabissa, Manna, Badiale, Riva, Castelnuovo and Molinari, 2020. On the leadership and management levels, long term focus should be on improving the workforce awareness on infectious diseases, increasing the nurses to patients' ratio, and expanding the promotional and reward systems to encourage and motivate the nurses.

\section{IMPLICATIONS AND LIMITATIONS}

The integrated literature review findings imply and emphasize cushion nurses' need during and after the Covid-19 pandemic. Also, it is evident that when recruiting and allocating nurses to the front-line areas, a consideration beyond skills and experience should be made. While as it is imperative to consider skills and experience, it is also relevant that nursing staff managers and hospitals should consider individual nurses well-being (Yifan, Ying, Chunhong, Jing, Rong, Zhenyu, Zejuan and Peihung, 2020; Giusti, Pedroli, D'Aniello, Badiale, Pietrabissa, Manna, Badiale, Riva, Castelnuovo and Molinari, 2020). Mental health stability and past exposures should be additional evaluation criteria. In the future, besides professional training on handling infectious diseases, further training and evaluation program on mental health on stability, memory loss, and concentration capacity, is critical. This is to help cushion and protect nurses with past traumatic experiences amidst infectious pandemics (Spoorthy, Pratapa and Mahant, 2020; Shah, Kamrai, Mekala, Mann, Desai and Patel, 2020). Further, offering such psychological support will protect and serve as a long term recovery plan and strategy for the nurses serving mainly as front line healthcare workers. Additionally, the study findings indicate the need to re-evaluate fatigue mitigation strategies. Nurses' fatigue levels have increased significantly in the wake of the Covid-19 pandemic. This is due to a lack of sufficient skills and experience, a lack of enough workforce, and the risk and increasing nurses' infection rates. In the future, strategies such as additional staffing, training, and nurses' educational program shifts should be implemented (Spoorthy, Pratapa and Mahant 2020; Di Tella, Romeo, Benfante and Castelli, 2020). The new orientation in training and development should empower the nurses' mental health as a way of strengthening and increasing their resolve and resilience in dealing with related future challenges.

Although the integrated literature review delivered on its aim, a significant challenge in the review paper was data availability. Although the pandemic has spread across the globe since December 2019, the lack of empirical and primary data on nurses' implications remains challenging. To this end, it sourced and analyzed data, both qualitative and quantitative, from three leading nursing databases. Future studies should be based on collecting primary empirical data through a longitudinal study. This would help indicate the fatigue levels and the mental health implications the Covid-19 pandemic has. This should be through comparing the fatigue levels for the front-line workers and the ordinary ward nurses.

\section{CONCLUSION}

Analysis of the Covid-19 pandemic impact on nurses' fatigue indicates a positive relationship. With the increased spread, Covid-19 has exposed nurses to an increasing workload exposing them to acute and chronic fatigue. Furthermore, the 
lack of enough nursing skills in handling infectious diseases exposes them to mental fatigue. Nurses with pre-existing mental conditions have a higher pre-disposition to fatigue. However, nurses have played an essential role as front-line workers in managing and caring for Covid-19 patients. Although the professional training and skills have enabled nurses to manage the pandemic with minimal casualties, it has also taken a toll on the profession itself. Increased workload, rising infection rates among nurses and mental health complications are among the leading challenges. The profession should develop a long-term strategy that includes increasing the nurses to patients' ratio and a long-term mental health and fatigue mitigation strategy.

\section{REFERENCES}

[1] Alharbi, J., Jackson, D. and Usher, K., 2020. The potential for COVID-19 to contribute to compassion fatigue in critical care nurses. Journal of Clinical Nursing

[2] Allen, C.S., Chen, Q., Willson, V.L. and Hughes, J.N., 2009. Quality of research design moderates effects of grade retention on achievement: A meta-analytic, multilevel analysis. Educational Evaluation and Policy Analysis, 31(4), pp.480-499

[3] Al-Masaeed, M.A.M., Al-Motiry, A., ALSABABHA, R.W.M., Alqudah, M. and Ismail, K., 2020. Investigating nursing staff experiences and experts recommendations evolution as Covid-19 infection curve flattens globally: an integrated literature Review IOSR Journal of Nursing and Health Science (IOSR-JNHS) e-ISSN: 2320-1959.p- ISSN: 2320-1940 Volume 9, Issue 5 Ser. VI (Sep. - Oct. 2020), PP 32-37

[4] Bennett, C.L., James, A.H. and Kelly, D., 2020. Beyond tropes: towards a new image of nursing in the wake of Covid-19. Journal of Clinical Nursing

[5] Blake, H., Bermingham, F., Johnson, G. and Tabner, A., 2020. Mitigating the psychological impact of COVID-19 on healthcare workers: a digital learning package. International Journal of Environmental Research and Public Health, 17(9), p.2997

[6] Cucinotta, D. and Vanelli, M., 2020. WHO declares COVID-19 a pandemic. Acta Bio Medica: Atenei Parmensis, 91(1), p.157.

[7] Dean, M and Gentry, E., 2020. Team-Based Care: Understanding Compassion Fatigue and Burnout in Navigation, AONN

[8] Di Tella, M., Romeo, A., Benfante, A. and Castelli, L., 2020. Mental health of healthcare workers during the COVID-19 pandemic in Italy. Journal of evaluation in clinical practice, 26(6), pp.1583-1587

[9] Fowler, K. and Wholeben, M., 2020. COVID-19: Outcomes for trauma-impacted nurses and nursing students. Nurse Education Today

[10] Giusti, E.M., Pedroli, E., D'Aniello, G.E., Badiale, C.S., Pietrabissa, G., Manna, C., Badiale, M.S., Riva, G., Castelnuovo, G. and Molinari, E., 2020. The psychological impact of the COVID-19 outbreak on health professionals: a cross-sectional study. Frontiers in Psychology, 11.

[11] Greenberg, N., Docherty, M., Gnanapragasam, S. and Wessely, S., 2020. Managing mental health challenges faced by healthcare workers during covid-19 pandemic. $b m j, 368$

[12] Hertel, R., 2020. The Use of Psychological PPE in the Face of COVID-19, AMSN

[13] Ho, W., 2008. Integrated analytic hierarchy process and its applications-A literature review. European Journal of operational research, 186(1), pp.211-228

[14] Hu, Y., Wang, L., Hu, S. and Fang, F., 2020. Facility-Level Case Report of Nursing Care Processes for Patients With Suspected 2019 Novel Coronavirus Disease in Shanghai, China. Journal of Emergency Nursing

[15] Kemerer, M., 2020. Secondary Traumatic Stress and Nurse Resiliency in the Face of the COVID-19 Pandemic, The Maryland Nurse Journal

[16] Kohtala, C., 2015. Addressing sustainability in research on distributed production: an integrated literature review. Journal of Cleaner Production, 106, pp.654-668

[17] Liu, Q., Luo, D., Haase, J.E., Guo, Q., Wang, X.Q., Liu, S., Xia, L., Liu, Z., Yang, J. and Yang, B.X., 2020. The experiences of healthcare providers during the COVID-19 crisis in China: a qualitative study. The Lancet Global Health.

[18] Mogi, R. and Spijker, J., 2020. The influence of social and economic ties to the spread of COVID-19 in Europe
[19] Nelson, S.M. and Lee-Winn, A.E., 2020. The mental turmoil of hospital nurses in the COVID-19 pandemic. Psychological Trauma: Theory, Research, Practice, and Policy

[20] Ng, Q.X., De Deyn, M.L.Z.Q., Lim, D.Y., Chan, H.W. and Yeo, W.S., 2020. The wounded healer: A narrative review of the mental health effects of the COVID-19 pandemic on healthcare workers. Asian journal of psychiatry

[21] Pfefferbaum, B. and North, C.S., 2020. Mental health and the Covid-19 pandemic. New England Journal of Medicine

[22] Pillai, S., Siddika, N., Apu, E.H. and Kabir, R., 2020. COVID-19: Situation of European countries so far. Archives of medical research

[23] Puhan, M.A., Schünemann, H.J., Murad, M.H., Li, T., Brignardello-Petersen, R., Singh, J.A., Kessels, A.G. and Guyatt, G.H., 2014. A GRADE Working Group approach for rating the quality of treatment effect estimates from network meta-analysis. Bmj, 349, p.g5630

[24] Ruktanonchai, N.W., Floyd, J.R., Lai, S., Ruktanonchai, C.W., Sadilek, A., Rente-Lourenco, P., Ben, X., Carioli, A., Gwinn, J., Steele, J.E. and Prosper, O., 2020. Assessing the impact of coordinated COVID-19 exit strategies across Europe. Science, 369(6510), pp. $1465-1470$

[25] Shah, K., Kamrai, D., Mekala, H., Mann, B., Desai, K. and Patel, R.S., 2020. Focus on mental health during the coronavirus (COVID-19) pandemic: applying learnings from the past outbreaks. Cureus, 12(3)

[26] Shim, E., Tariq, A., Choi, W., Lee, Y. and Chowell, G., 2020. Transmission potential and severity of COVID-19 in South Korea. International Journal of Infectious Diseases

[27] Spoorthy, M.S., Pratapa, S.K. and Mahant, S., 2020. Mental health problems faced by healthcare workers due to the COVID-19 pandemic-A review. Asian journal of psychiatry, 51, p.102119

[28] Spoorthy, M.S., Pratapa, S.K. and Mahant, S., 2020. Mental health problems faced by healthcare workers due to the COVID-19 pandemic-A review. Asian journal of psychiatry, 51, p.102119.

[29] Sun, N., Wei, L., Shi, S., Jiao, D., Song, R., Ma, L., Wang, H., Wang, C., Wang, Z., You, Y. and Liu, S., 2020. A qualitative study on the psychological experience of caregivers of COVID-19 patients. American journal of infection control, 48(6), pp.592-598

[30] Sun, N., Wei, L., Shi, S., Jiao, D., Song, R., Ma, L., Wang, H., Wang, C., Wang, Z., You, Y. and Liu, S., 2020. A qualitative study on the psychological experience of caregivers of COVID-19 patients. American journal of infection control, 48(6), pp.592-598

[31] Yifan, T., Ying, L., Chunhong, G., Jing, S., Rong, W., Zhenyu, L., Zejuan, G. and Peihung, L., 2020. Symptom Cluster of ICU nurses treating COVID-19 pneumonia patients in Wuhan, China. Journal of Pain and Symptom Management 